\title{
TYPOLOGIE DES MOLLUSQUES DES EAUX COURANTES. ORGANISATION BIOTYPOLOGIQUE ET GROUPEMENTS SOCIOECOLOGIQUES
}

\author{
par J. MOUTHON1
}

\begin{abstract}
Une analyse statistique de 59 espèces ou formes (pisidies) de Mollusques dulcicoles, effectuée sur 91 stations jalonnant 9 cours d'eau $(1060 \mathrm{~km})$ a montré l'existence d'une structure malacologique théorique formée d'une succession d'espèces pouvant s'inscrire dans 10 noyaux.

Une étude de la distribution de chaque espèce permet d'apprécier :

- son preferendum et son amplitude typologique, reliés à son degré d'eurytopie et d'euryécie,

- de classer chacune d'elle à l'intérieur de groupements "socioécologiques",

- de comparer l'évolution générale des espèces de Mollusques à 4 groupes faunistiques ayant fait antérieurement l'objet des mêmes études.
\end{abstract}

\section{Typology of molluscs of flowing water - biotypological organisations - socioecological groupings.}

A statistical analysis of 59 species or forms (pisidies) of freshwater molluscs from 91 stations and 9 watercourses $(1.060 \mathrm{~km})$ demonstrated a theoretical malacological structure formed by a succession of species that could be recorded in 10 nuclei.

A study of the distribution of each species has shown:

- its preferendum and typological range, partially dependent on its degree of eurytopy and euryoecy,

- that each one can be vlaced in a "socioecological" group,

- that a comparison can be made within the general evaluation of mollusc species with four faunistic groups that have been studied previously.

\section{1. - INTRODUCTION}

Dès 1935, Zhadin définit des "groupements d'espèces " associés à différents types d'écosystèmes et de milieux aquatiques : sources. ruisseaux de montagne, rivières de plaines, lacs, étangs... et en 1936, Boycott publie la première synthèse écologique concernant les Mollusques d'eau douce. Depuis, il faut bien convenir que peu de progrès

1. Laboratoire d'Hydrobiologie du C.E.M.A.G.R.E.F. Institut des Sciences Naturelles, 25000 Besançon. 
ont été effectués dans ce domaine, les travaux actuels étant surtout de nature descriptive, réalisés plus souvent dans les milieux lacustres que dans les eaux courantes et concernant plus fréquemment la biologie des espèces que leur écologie.

Les études de cours d'eau visant à établir une classification biologique des écosystèmes d'eau courante, permettent d'avoir une idée assez précise de la distribution longitudinale et de l'importance du phénomène de vicariance chez certains groupes faunistiques comme les Poissons (Huet 1949), les Plécoptères, les Ephéméroptères, les Trichoptères, les Hydracariens... (cf. Mouthon 1979).

Toutefois, en ce qui concerne les Mollusques, trop souvent négligés dans les recherches hydrobiologiques ou étudiés seulement au niveau de cours d'eau de faibles dimensions ou de secteurs de rivières d'importance modeste (Mouthon 1979), les documents proposés ne permettent pas d'établir la répartition écologique des Mollusques dans l'écosystème "eau courante" ni de proposer des groupements écologiques d'espèces.

Nous avons recherché, au moyen d'une méthode mathématique, l'existence d'une distribution longitudinale théorique des espèces de Mollusques dans un réseau hydrographique constitué par 9 cours cours d'eau et considéré comme une unité fonctionnelle.

\section{2. - MATERIEL ET METHODES}

Les caractéristiques géologiques, hydrologiques, physico-chimiques des 9 cours d'eau retenus pour la réalisation de ces recherches ont été décrites en détail dans les travaux de Verneaux (1973) et Mouthon (1979-1980). L'ensemble de ces écosystèmes constitué par le Doubs, 6 de ses affluents, l'Ognon et l'Aube, forme un réseau hydrographique de $1060 \mathrm{~km}$ et drainent un bassin versant d'environ $15850 \mathrm{~km}^{2}$ (fig. 1).

Les techniques d'échantillonnage ont permis le recensement de 30775 individus comprenant 59 espèces ou formes (Tableau I) dont l'abondance sur chaque station figure dans une matrice des données (cf. Mouthon, 1980). La méthode de traitement des données utilisées : l'analyse factorielle des correspondances et ses applications aux écosystèmes d'eau courante, ont été exposées et décrites lors de précédents travaux (Cordier 1965...). 


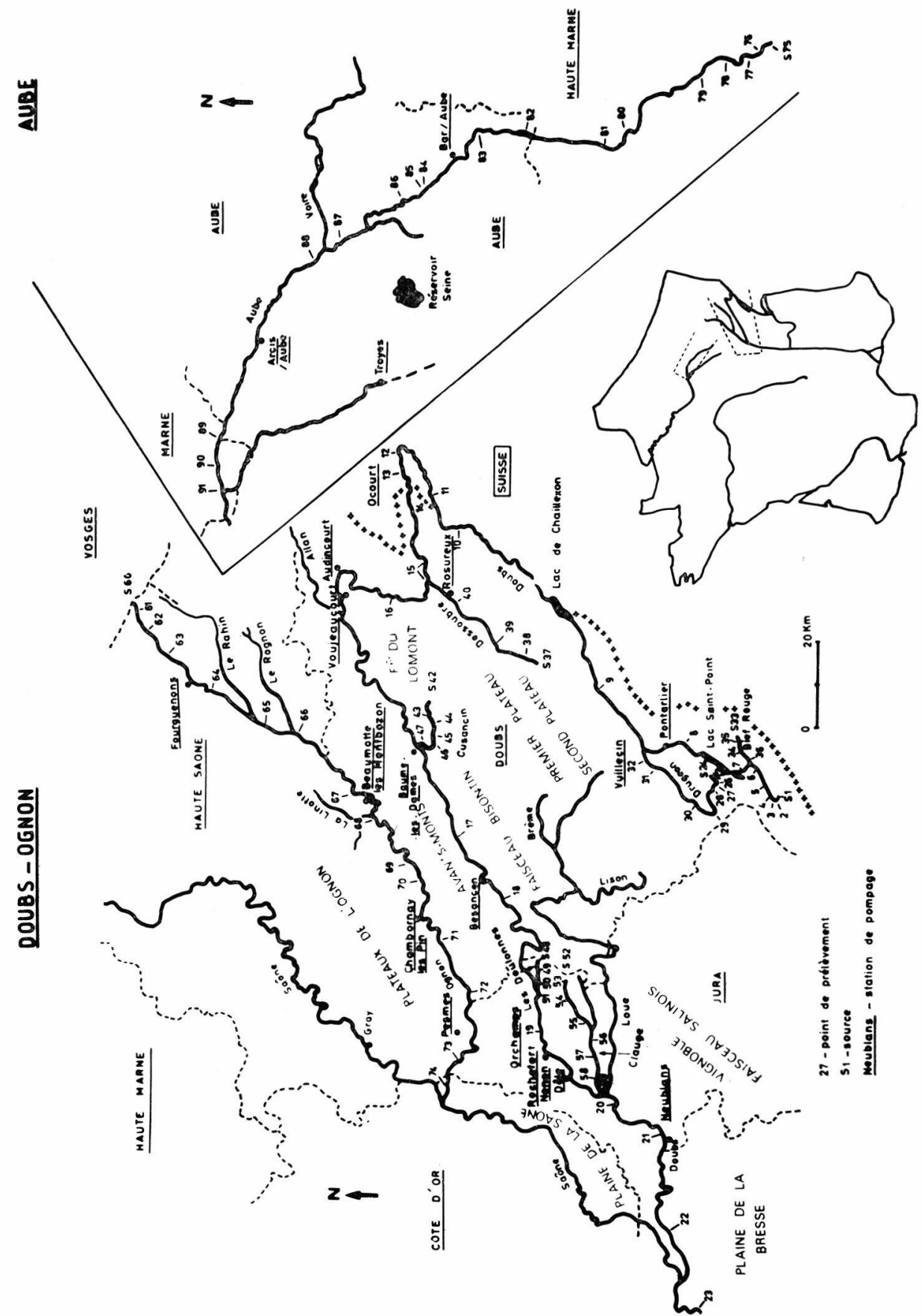

Fig. 1. - Réseaux hydrographiques du Doubs, de l'Ognon et de l'Aube, répartition des stations d'étude. 
TABlEaU I. - Liste des espèces de Mollusques et des abréviations utilisées dans le graphique représentant la structure malacologique du réseau hydrographique étudié.

Hauffenia minuta - Draparnaud, 1805 $(\mathrm{Hm})$

Bythinella viridis - Poiret, 1801 (Bv)

Bythiospeum diaphanum - Michaud, 1831 (Bd)

Bythinella carinulata - Drouet, 1867 (Ba)

Bythinella dunkeri - Von Frauenfeld, 1856 (Bi)

Pisidium personatum - Malm, 1855 (Pp)

Anisus spirorbis - Linné, 1758 (As)

Galba truncatula - Müller, 1774 (Gt)

Pisidum obtusale - Lamarck, 1818 (Po)

Pisidium casertanum - Poli, 1791 (Pe)

Ancylus fluviatilis - Müller, 1774 (Af)

Radix peregra - Müller, 1774 (Rp)

Pisidium amnicum - Müller, 1774 (Pi)

Pisidium milium - Held, 1836 (Pg)

Bathyomphalus contortus - Linné, 1758 (Bc)

Pisidium pulchellum - Jenyns, 1832 (Py)

Pisidium hibernicum - Westerlund, $1894(\mathrm{Ph})$

Pisidium nitidum - Jenyns, 1932 (Pu)

Pisidium subtruncatum - Malm, 1855 $(\mathrm{Pb})$

Pisidium tenuilineatum - Stelfox, 1918 (Pn)

Galba palustris - Müller, 1774 (Gp)

Sphaerium corneum - Linné, 1758 (So)

Planorbis carinatus - Müller, 1774 (Pc)

Physa fontinalis - Linné, 1758 (Pf)

Anisus vortex - Linné, 1758 (Av)

Valvata piscinalis - Müller, $1774(\mathrm{Vp})$

Valvata cristata - Müller, 1774 (Vc)

Gyraulus albus - Müller, 1774 (Ga)

Pisidium henslowanum - Sheppard, 1823 (Pw)
Bithynia tentaculata - Linné, 1758 (Bt)

Lymnaea stagnalis - Linné, 1758 (Ls)

Unio crassus - Philipsson, 1788 (Uc)

Myxas glutinosa - Müller, 1774 (Mg)

Acroloxus lacustris - Linné, 1758 (Al)

Theodoxus fluviatilis - Linné, 1758 (Tf)

Bithynia leachi - Sheppard, 1823 (Bl)

Hippeutis complanata - Linné, 1758 (Hc)

Pseudanodonta complanata - Rossmassler, 1835 (Pl)

Pisidium moitessierianum - Paladilhe, 1866 (Pm)

Pisidium supinum - Schmidt, 1851 (Ps)

Planorbarius corneus - Linné, 1758 (Pr)

Potomida littoralis - Cuvier, $1797(\mathrm{Pt})$

Radix auricularia - Linné, 1758 (Ra)

Sphaerium lacustre - Müller, 1774 (Sl)

Armiger crista - Linné, 1758 (Ac)

Anodonta anatina - Linné, 1758 (Aa)

Unio pictorum - Linné, 1758 (Up)

Potamopyrgus jenkinsi - Smith, 1889 (Pi)

Viviparus viviparus - Linné, 1758 (Vv)

$P$. casertanum f. ponderosa - Stelfox, $1918(\mathrm{Pd})$

P. nitidum f. crassa - Stelfox, 1918 (Pk)

Physa acuta - Draparnaud, 1805 (Pa)

$P$. subtruncatum f. incrassata - Stelfox, $1918(\mathrm{Pz})$

Sphaerium rivicola - Lamarck, 1818 (Sr)

Unio tumidus - Philipsson, 1788 (Ut)

Ferrissia wautieri - Mirolli, 1960 (Fw)

Dreissena polymorpha - Pallas, 1771 (Dp)

Sphaerium solidum - Normand, 1844 (Ss)

Segmentina nitida - Müller, 1774 (Sm)

\section{3. - RESULTATS : \\ ANALYSE GLOBALE DU RESEAU HYDROGRAPHIQUE ETUDIE}

\subsection{Structure suivant les axes $F_{1} F_{2}$}

La distribution du couple espèces - stations dans le plan des deux premiers axes représentant $22 \%$ d'inertie expliquée s'effectue en trois groupements, exception faite d'une espèce ( $P$. obtusale) dont la posi- 
tion périphérique, due à sa faible fréquence, témoigne de son caractère aléatoire (fig. 2).

Les groupements I et II nettement individualisés sont représentatifs de la zone des sources et du secteur aval immédiat.

Le groupement III représentant l'essentiel de la structure malacologique du réseau s'organise en une succession continue des espèces ( continuum ") sans groupement bien individualisé. La contribution aux axes et notamment à l'axe 2 de toutes les espèces est nulle ou voisine de 0 , l'essentielle de celle-ci étant assurée par les espèces des sources. Ceci confirmerait la signification de l'axe 1 considéré comme exprimant la "complexité croissante» du milieu par Masonie et Al. (1971) ou son " évolution progressive » ainsi que celle des peuplements (Verneaux 1973).

La plupart des espèces, hormis celles du secteur des sources, s'organisant presque exclusivement en fonction de l'axe de "succession " $F_{1}$, nous avons été conduit à étudier d'autres combinaisons d'axes susceptibles de dissocier cet alignement.

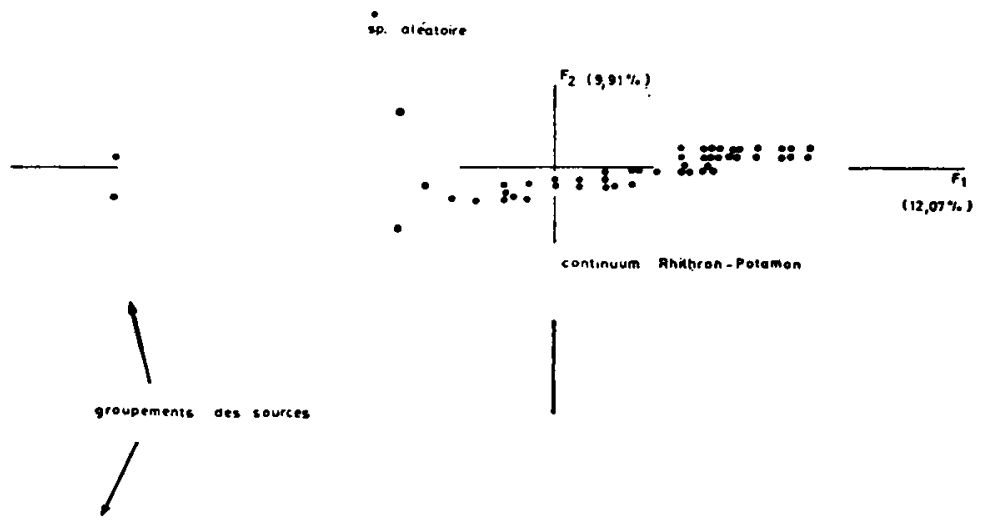

Frg. 2. - Structure malacologique du réseau hydrographique étudié dans le plan $F_{1} F_{2}$ (stations non représentées).

\subsection{Structure suivant les axes $\mathbf{F}_{1} \mathbf{F}_{3}$}

La répartition des espèces dans le plan des axes $F_{1} F_{3}$ représentant $21,6 \%$ de l'inertie expliquée, soit approximativement la même que pour les axes $F_{1} F_{2}$, s'organise suivant un continuum a noda où 11 groupements d'espèces ou noyaux peuvent être distingués (fig. 3 ). 


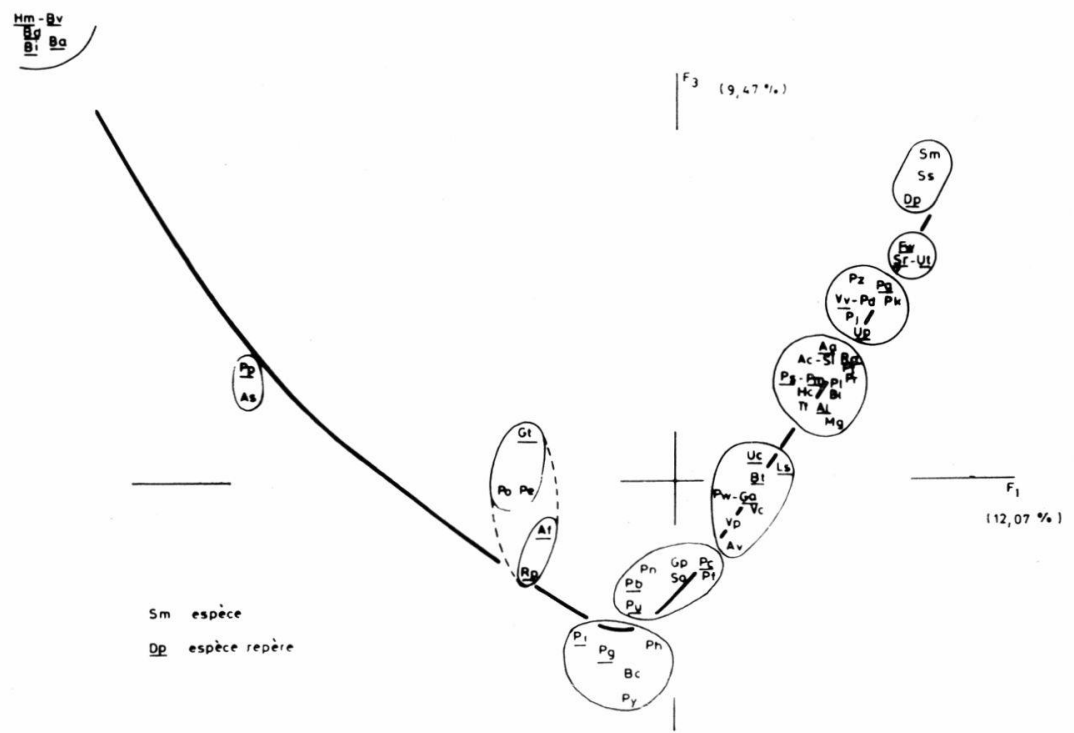

Fig. 3. - Structure malacologique du réseau hydrographique étudié dans le plan $\mathrm{F}_{1} \mathrm{~F}_{3}$ (stations non représentées).

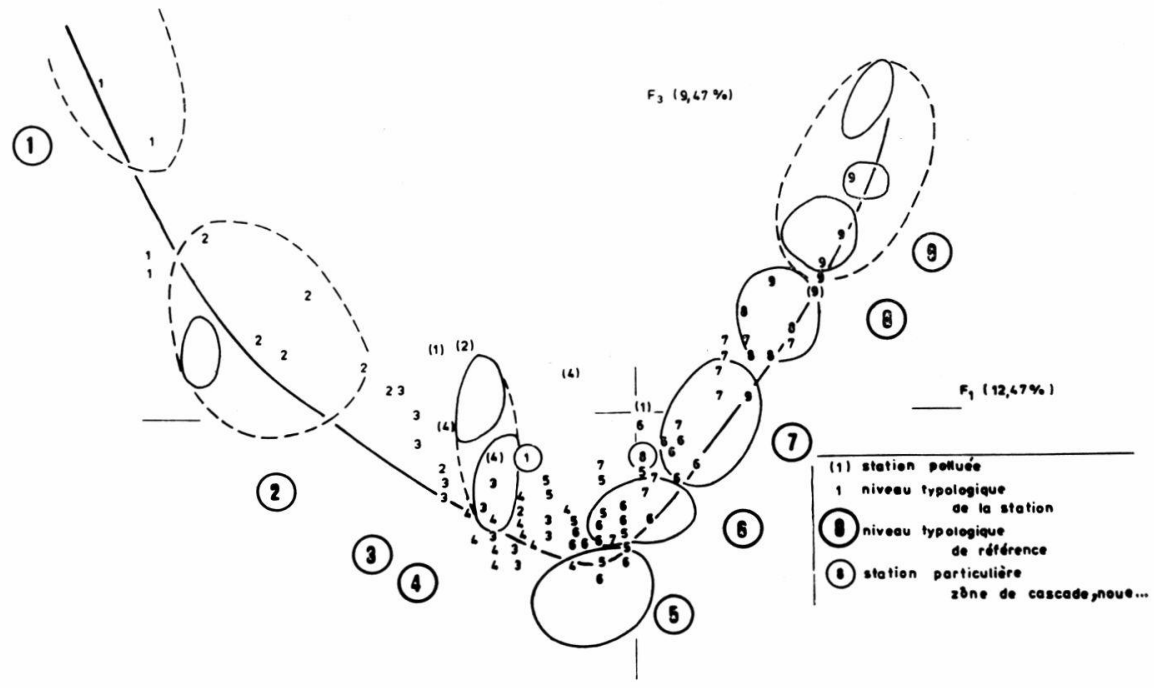

FIG. 4. - Distribution des stations d'études (représentées par leur niveau typologique) et des groupements d'espèces.

Les travaux permettant de définir différentes "zones » ou plutôt différents niveaux se succédant le long d'un écosystème d'eau courante se réduisent essentiellement en Europe : 
- aux classifications zonales proposées par Huet (1949) où pente et largeur du cours d'eau sont les paramètres dominants puis, par Illies et Botosaneau (1963) pour lesquels ce sont les confluences d'égale importance et par conséquent le débit qui joue le rôle prépondérant ;

- aux niveaux typologiques définis statistiquement par l'intermédiaire de groupements d'espèces proposés par Verneaux (1973-1976 b et 1977 a) ou de paramètres écologiques (Verneaux 1977 b).

\subsection{Confrontation de la structure malacologique obtenue aux données typologiques : typologie des Mollusques}

Dans le plan des axes $F_{1} F_{3}$ nous avons substitué aux repères des stations leur appartenance typologique et projeté sur le même graphique (fig. 4) à leurs emplacements respectifs, les groupements d'espèces précédemment délimités (fig. 3) permettant d'associer d'une manière satisfaisante ces derniers aux niveaux typologiques proposés.

La liste des espèces composant les différents noyaux est donnée dans l'ordre de la succession typologique. Les espèces qui possèdent à l'intérieur de chaque groupement les plus fortes contributions aux axes sont considérées comme les repères de l'organisation malacologique, dans l'espace abstrait, d'un écosystème synthétique intégrant les peuplements des différents cours d'eau étudiés. Elles sont soulignées (fig. 3) et répertoriées dans le tableau II.

Niveau $1:$ ( 6 espèces: 4 Prosobranches, 1 Bivalves) : H. minuta, $B$. diaphanum, $B$. viridis, $B$. carinulata, B. dunkeri, $P$. personatum. La fréquence des 5 premières espèces étant très faible, elles occupent sur le graphique des positions périphériques. En revanche, $P$. personatum, dont la présence au niveau des sources en association avec différentes espèces de Bythinella est remarquablement constante, occupe une position plus structurale. A. spirorbis est une espèce de milieux aquatiques susceptibles de s'assécher périodiquement (ruisseaux, mares). Ces milieux n'ayant pas été systématiquement prospectés ici, elle se trouve en compagnie de $P$. personatum dans le seul cours d'eau de cette nature inventorié (Drugeon supérieur $=$ Bief Belin).

Cn trouve parfois quelques exemplaires de $P$. personatum dans certains prélèvements effectués loin des sources. Dans ce cas, une étude plus détaillée des habitats de la station montre que l'espèce est toujours très localisée et provient pour la majorité des situations d'afférences latérales, cependant pour de rares cas, la présence de cette pisidie pourrait résulter de l'existence de sources dans le lit même de la rivière.

Une étude récente effectuée sur le Gapeau (Var) a montré que cette 
espèce était présente des sources à la mer, or plusieurs sources noyées ont été mises en évidence dans le lit de cette petite rivière côtière.

Niveau 2: il n'existe pas dans le réseau hydrographique étudié d'espèces directement associées à ce niveau, sauf peut être G. truncatula dont la tendance crénophile et le caractère amphibie ont déjà été soulignés précédemment (Mouthon 1979).

Seule l'autécologie de ce Gastéropode permettra de préciser son appartenance à un niveau donné.

Dans ce groupement, deux pisidies lui sont associées: $P$. obtusale est recensée seulement au voisinage des sources et du cours supérieur de la Clauge (forêt de Chaux) dans les sédiments de flaques en voie d'assèchement dont l'eau, déjà peu minéralisée, est plus ou moins acidifiée par les substances humiques et les feuilles en décomposition. D'après Kuiper (1966), cette espèce évite les cours d'eau et préfère les eaux stagnantes, peu profondes et plutôt acides.

$P$. casertanum, en revanche, est très largement répandue et colonise tous les milieux aquatiques même temporaires (Kuiper 1966).

Ces trois espèces associées à $A$. spirorbis et $A$. hypnorum, non recensée sur le réseau étudié, constituent un groupement caractéristique des milieux temporaires (mares, ruisseaux de drainage, fossés...).

$G$. truncatula et $P$. casertanum susceptibles, comme l'indique la matrice des résultats bruts, d'être présentes des niveaux 1 à 9 peuvent être qualifiées d'espèces euryèces. Toutefois, la présence fréquente de ces deux Mollusques, à substrat riche en matières organiques (Ognon, Drugeon), n'ayant pas un caractère de résurgence, ainsi que leur présence sporadique au niveau de l'ensemble du cours d'eau (Rhithron et Potamon) donne à ce noyau, pourtant typiquement euryèce, une position assez éloignée de l'origine des axes.

Niveaux (2), 3 et $4:$ ( 2 espèces : 2 Pulmonés basommatophores), $A$. fluviatilis et $R$. peregra peuvent être associées aux niveaux 2 à 4 sur lesquels ce sont souvent les deux seules espèces présentes. Il apparaît, comme dans le groupement précédent, que la position de ces dernières sur le graphique ne constitue pas directement une conséquence de leur présence (l'amplitude typologique d'A. fluviatilis allant de 1 à 9 ; celle de $R$. peregra de 1 à 7) mais plutôt de l'absence d'autres espèces à ces niveaux.

Niveau $5:$ ( 5 espèces : 1 Pulmoné basommatophore et 4 Bivalves), $P$. amnicum, $P$. milium, $P$. hibernicum, $B$. contortus, $P$. pulchellum. $P$. amnicum le plus gros des Pisidium $(11 \times 9 \mathrm{~mm})$ est fréquemment associé à la présence d'épaisses zones sédimentaires riches en matières organiques sur lesquelles coulent des eaux bien oxygenées. $P$. hibernicum, que l'on trouve en abondance dans les lacs (St Point et 
Remoray, les Rousses...) n'est pas « une espèce essentiellement lacustre " (Germain 1931) ; elle vit également dans d'autres milieux, notamment les caneaux et les rivières mais toujours en très faible abondance (Kuiper 1966).

$P$. pulchellum, espèce " rare des plaines de moyenne latitude " (Kuiper 1966) n'a été recensée qu'au niveau du Drugeon moyen et inférieur à $820 \mathrm{~m}$ d'altitude. Sa position plutôt périphérique résulte sans doute de sa faible fréquence ( 3 stations).

Nota: Au cours d'un échantillonage récent des émissaires du lac des Rousses nous avons à nouveau découvert cette espèce dans le cours inférieur du Bief Noir ainsi que dans le lac lui-même au niveau du confluent dont l'altitude avoisine $1060 \mathrm{~m}$.

Niveau 6 : (7 espèces : 3 Pulmonés basommatophores et 4 Bivalves), $P$. nitidum, P. subtruncatum, $P$. tenuilineatum, S. corneum, G. palustris, $P$. carinatus, $P$. fontinalis. Dans leur aire de répartition commune $P$. fontinalis et $P$. acuta sont vicariantes. L'amplitude typologique de la dernière espèce est faible ( 8 à 9). Dans le sud de la France, seule $P$. asuta est représentée, elle atteint alors son degré d'euryécie maximale (amplitude -ypologique 6 à 9), observations effectuées sur le bassin de la Dordogne, le Gapeau et le torrent de Parma en Italie (cf. Odum, 1976).

Niveau 7 : ( 8 espèces : 3 Pulmonés basommatophores, 3 Prosobranches, 2 Bivalves), A. vortex, $V$. piscinalis, $V$. cristata, $P$. henslowanum, $G$. albus, B. tentaculata, L. stagnalis, U. crassus. La position subcentrale et la faible contribution aux axes des espèces de ce noyau traduisent le caractère euryèce de ce groupement qui, compte tenu de ia distribution particulière des Mollusques le long de la structure (les $3 / 4$ des espèces sont réparties entre les niveaux 5 et 9) fait figure de noyau de transition entre Rhithron et Potamon (Ecotone).

Niveau 8 : (14 espèces : 5 Pulmonés basommatophores, 2 Prosobranches, 7 Bivalves), $M$. glutinosa, $T$. fluviatilis, $A$. lacustris, $H$. complanata, B. leachi, $P$. moitessierianum, $P$. supinum, $P$. complanata, $S$. lacustre, $A$. crista, $P$. corneus, $P$. littoralis, $R$. auricularia et $A$. anatina.

$M$. glutinosa est une espèce de plaine qui évite les zones montagneuses (Germain 1931) ; elle n'a été recensée qu'au niveau du cours inférieur de l'Aube et c'est dans les noues que son abondance est maximale.

$P$. complanata est cocénotique d'A. anatina, $P$. littoralis, $U$. pictorum et à certains niveaux (Rhithron inférieur et Potamon) d'U. crassus mais n'est jamais découverte en grande abondance et semble se cantonner dans les eaux profondes.

S. lacustre vit dans les milieux d'eau calme, peu profonds, à végé- 
tation souvent dense et à vase riche en matières organiques (Favre 1927 ; Kuiper et Wolff 1970 ; Meir-Brook 1970 ; Walter et Kuiper 1978); sa présence est constante dans les noues du Doubs inférieur.

Niveau 9 : (13 espèces : 3 Pulmonés basommatophores, 2 Prosobranches, 8 Bivalves), $U$. pictorum, $P$. jenkinsi, $V$. viviparus, $P$. casertanum f. ponderosa, $P$. nitidum f. crassa, $P$. acuta, $S$. rivicola, $U$. tumidus, $F$. wautieri, $D$. polymorpha, $S$. solidum, $S$. nitida, $P$. substruncatum $\mathrm{f}$. incrassata. On trouve $P$. jenkinsi dans les cours inférieurs du Doubs, de l'Aube et dans la Saône au niveau de la confluence Doubs-Saône, mais dans des abondances notablement faibles qui n'ont rien de comparables à celles mentionnées dans la littérature: 10000 ind $/ \mathrm{m}^{2}$ (Macan, 1950), $30000 \mathrm{ind} / \mathrm{m}^{2}$ (Adam, 1942 - Heywood et Edward, 1962) ou que j'ai pu observer moi-même dans le cours supérieur de l'Amance (affluent de l'Aube), de la Seille (niveau typologique 4-5) ou du Gapeau. De ce fait, son preferendum typologique pourrait être plus apicale (niveaux 6-7 ?). Toutefois, si la présence de cette espèce parthénogénétique est fréquente dans le cours inférieur des grandes rivières prospectées (sauf l'Ognon), il n'en est pas de même dans les secteurs supérieurs où elle est très sporadique et son abondance variable peut passer de quelques individus à une centaine de mille d'une année à l'autre puis disparaître totalement.

Les pisidies a fortes charnières (formes pondéreuses) munies d'un appendicule comme $P$. supinum, $P$. moitessierianum ou seulement à charnières épaissies comme les variétés de $P$. casertanum $\mathrm{f}$. ponderosa, $P$. nitidum f. crassa, $P$. subtruncatum $\mathrm{f}$. incrassata apparaissent comme caractéristiques du Potamon.

Seule la très petite espèce $P$. tenuilineatum $(2 \times 1,5 \mathrm{~mm})$ semble faire exception à cette règle puisqu'elle appartient au niveau 6 (Rhithron inférieur), il semble cependant que les individus des niveaux inférieurs aient une charnière plus épaisse que le "type " qui prolifère à partir du Rhithron moyen (niveau 4-5).

$F$. wautieri souvent confondue avec A. lacustris est abondante dans toute la Saône moyenne et inférieure où Calas (1954) l'avait déjà échantillonnée mais aussi au niveau des stations ultimes de l'Ognon et du Doubs inférieur où elle est plus rare. Il est intéressant de constater que toutes les Ferrissia rencontrées en rivière et dans les noues appartiennent exclusivement au type ancylö̈de, aucune forme septifère n'ayant été découverte.

\section{REMARQUe: VÉRIFICATION DES GROUPEMENTS D'ESPÈCES}

Le traitement des données au moyen d'une analyse numérique ascendante hiérarchisée portant sur les 7 premiers facteurs et représentant $52,8 \%$ de l'inertie expliquée, a permis de vérifier l'exactitude des groupements proposés. Toutefois, il est à remarquer que ce type de traite- 
ment ne peut être utilisé seul dans le cadre d'études biotypologiques, puisque l'énumération des noyaux ne s'effectue plus suivant la structure définie par les axes $\mathrm{F}_{1} \mathrm{~F}_{2}$ ou $\mathrm{F}_{1} \mathrm{~F}_{3}$.

Tableau II. - Peuplement malacologique (espèces repères) des différents niveaux typologiques. (1) : propositions effectuées par Verneaux (1980).

\begin{tabular}{|c|c|c|c|c|}
\hline & ation & $\begin{array}{c}\text { Niveaux } \\
\text { typologiques } \\
\text { (Verneaux, }\end{array}$ & $\begin{array}{l}\text { Espèces } \\
\text { repères }\end{array}$ & $\begin{array}{l}\text { Contribution } \\
\text { aux axes }\end{array}$ \\
\hline Huet & Illies & & & \\
\hline Secteurs & EUCRÉNON & $\mathrm{B}_{0}$ & ? & \\
\hline & & & B. diaphanum & 149 \\
\hline & & & H. minuta & 130 \\
\hline & & & B. viridis & 217 \\
\hline & & $\mathrm{B}_{1}$ & B. carinulata & 111 \\
\hline & HYPOCRÉNON & & B. dunkeri & 100 \\
\hline & & & P. personatum & 168 \\
\hline & & $\mathrm{B}_{2}$ & G. truncatula & 25 \\
\hline sup. & EPIRHITHRON & $\mathrm{B}_{3}$ & $\begin{array}{l}\text { A. fluviatilis } \\
R . \text { peregra }\end{array}$ & $\begin{array}{l}36 \\
82\end{array}$ \\
\hline ZONE & & $\mathrm{B}_{4}$ & & \\
\hline SALMONICOLE & MÉTARHITHRON & $\mathrm{B}_{5}$ & $\begin{array}{l}\text { P. amnicum } \\
\text { P. milium }\end{array}$ & $\begin{array}{l}30 \\
51\end{array}$ \\
\hline moy. & & & P. nitidum & 62 \\
\hline & HYPORHITHRON & $\mathrm{B}_{6}$ & $\begin{array}{l}P . \text { subtruncatum } \\
P \text {. carinatus }\end{array}$ & $\begin{array}{l}49 \\
48\end{array}$ \\
\hline $\begin{array}{l}\text { inf. ou à } \\
\text { Ombre }\end{array}$ & & & G. albus & 7 \\
\hline & EPIPOTAMON & $\mathrm{B}_{7}$ & $\begin{array}{l}\text { B. tentaculata } \\
\text { L. stagnalis } \\
\text { U. crassus }\end{array}$ & $\begin{array}{r}10 \\
8 \\
6\end{array}$ \\
\hline sup. ou à & & & & \\
\hline Barbeau & & & A. lacustris & 25 \\
\hline & & & P. moitessierianum & 25 \\
\hline & MÉTAPOTAMON (1) & $\mathrm{B}_{8}$ & P. supinum & 22 \\
\hline & & & R. auricularia & 51 \\
\hline & & & A. anatina & 33 \\
\hline ZONE & & & & \\
\hline CYPRINICOLE & & & U. pictorum & 34 \\
\hline & & & V. viviparus & 44 \\
\hline & & & P. acuta & 38 \\
\hline inf. ou à & & $\mathrm{B}_{0}$ & S. rivicola & 45 \\
\hline Brème & HYPOPOTAMON (1) & & T. tumidus & 30 \\
\hline & & & F. wautieri & 38 \\
\hline & & & D. polymorpha & 29 \\
\hline
\end{tabular}




\section{4. - DISCUSSION}

Bien que faisant partie du même groupement défini dans un espace abstrait, les espèces de Bythinella recensées ne sont pas cocénotiques; en effet, conformément au principe de Monard, une seule espèce de ces petits Hydrobiidae est représentée dans chaque source. En outre, ce noyau ne semble pas exclusivement caractéristique du niveau $B_{1}$; une prospection récente du réseau hydrographique du Gapeau (Var) nous a permis de constater la présence de Bythinella parfois associée à Belgrandia dans des sources de niveau typologique 2 et 3 .

Des espèces comme A. fluviatilis, P. casertanum, G. truncatula reconnues comme euryèces et eurytopes par les échantillonnages effectués sur les Bassins du Doubs, de l'Aube, etc. et par de nombreuses données bibliographiques, peuvent avoir une position graphique assez éloignée de l'origine des axes, et des contributions à ceux-ci plutôt élevées, lorsqu'elles sont les seules espèces représentées au niveau du réseau supérieur de nombreuses rivières (niveau (1) 2-3-4). Dans ce cas, leur position n'est pas tant définie par leur présence sur un secteur donné mais bien plutôt par l'absence d'autres espèces représentatives des niveaux typologiques apicaux. Elles peuvent donc, dans une certaine mesure, servir à caractériser le Rhithron supérieur et moyen.

La hiérarchisation des espèces proposées par l'analyse factorielle des correspondances permet d'apprécier l'intensité de la vicariance au sein de l'Embranchement des Mollusques et on peut constater que celle-ci est d'autant plus importante que le genre considéré comprend d'espèces (cf. pisidies).

D'une manière générale, les pourcentages d'inertie représentant la participation des axes à l'explication de la distribution des données sont, si on les compare à ceux obtenus avec d'autres groupes faunistiques (Verneaux 1973), relativement faibles. On peut attribuer ceci à l'absence d'espèces électives des niveaux typologiques supérieurs (23-4) et au fait que les Mollusques occupant en majorité le faciès lénitique, n'intègrent qu'une partie de la structure d'un écosystème d'eau courante.

\section{5. - CLASSEMENT “ SOCIOLOGIQUE » DES ESPECES : EURYTOPIE, STENOTOPIE, EURYECIE, STENOECIE, PREFERENDUM TYPOLOGIOUE}

Chaque espèce se trouve associée à un noyau donné exprimant son preferendum écologique général. Par ailleurs un tableau de répartition des espèces mentionnant l'occurence de chacune d'elles au niveau 
des différentes stations classées selon leur niveau précise leur amplitude typologique, expression de leur degré d'eurytopie et d'euryécie (Tableau III).

On peut ainsi, en considérant ces deux critères, distinguer 3 groupes d'espèces :

- les plus sténotopes ou "espèces repères" ont une amplitude typologique faible ; $\Delta=3 \pm 1$. Parmi celles-ci certaines sont sténoèces et également sténothermes; c'est le cas pour les Hydrobiidae crénophiles appartenant aux genres Bythinella, Hauffenia, Bythiospeum... alors que d'autres espèces telles que $T$. fluviatilis, $F$. wautieri, $R$. auricularia, $V$. viviparus, $S$. rivicola, $U$. pictorum, A. anatina, $D$. polymorpha... sont euryèces. On constate donc, comme l'ont souligné Sacchi et Testard (1971) que la correspondance entre sténotopie et sténoécie n'est ni constante ni absolue ;

- des espèces intermédiaires ; $\Delta=6 \pm 1$;

- les plus eurytopes et euryèces à l'échelle du réseau considéré ont une position proche de l'origine des axes; $\Delta=9 \pm 1$.

La confrontation des données obtenues à la structure chevauchante composée de 12 groupements "socioécologiques " d'espèces proposée par Verneaux (1976 b) dont "l'organisation se compose à 3 degrés différents d'euryécie de courbes de Gauss successives exprimant des preferendum typologiques " (fig. 5) et définissant conformément à la nomenclature des phytosociologues (Guinochet, 1973) une classe $(\Delta=$ $9 \pm 1$ niveaux typologiques) 4 ordres $(\Delta=6 \pm 1)$ et 7 alliances $(\Delta=$ $3 \pm 1$ ) permet, dans ce cadre théorique, d'établir l'appartenance des Mollusques à 7 groupements socioécologiques : 1 classe, 2 ordres (II et IV), 4 alliances $(1,4,5,6)$ et 1 sous-alliance $(6 a)$.

Définis par Duvignaud (1946) comme étant « un ensemble d'espèces marquées par une tendance à se rassembler dans un biotope donné » ils sont ici déterminés dans un espace abstrait, les espèces de chaque groupement "socioécologique " n'étant pas obligatoirement cocénotiques. En effet, l'échantillonnage des espèces tel qu'il est réalisé par les phytosociologues nécessite pour l'ensemble des ordres d'invertébrés benthiques un temps beaucoup trop long pour qu'il soit entrepris sur un réseau hydrographique aussi vaste que celui étudié dans ce travail.

L'analyse du tableau III permet de constater que parmi les groupements d'ordre, un seul est d'ordre II alors que tous les autres sont d'ordre IV (amplitude typologique de 3 à 9), en ce qui concerne les alliances et ormis les espèces de sources; alliance 1 (niveau 0 à 3 ) on obtient une alliance $4(\Delta=3$ à 6$)$, une alliance $5(\Delta=4$ à 7$)$ et 5 alliances $6(\Delta=6$ à 9$)$ dont une sous-alliance, ce qui traduit bien le caractère potamophile des Mollusques dulçaquicoles. 


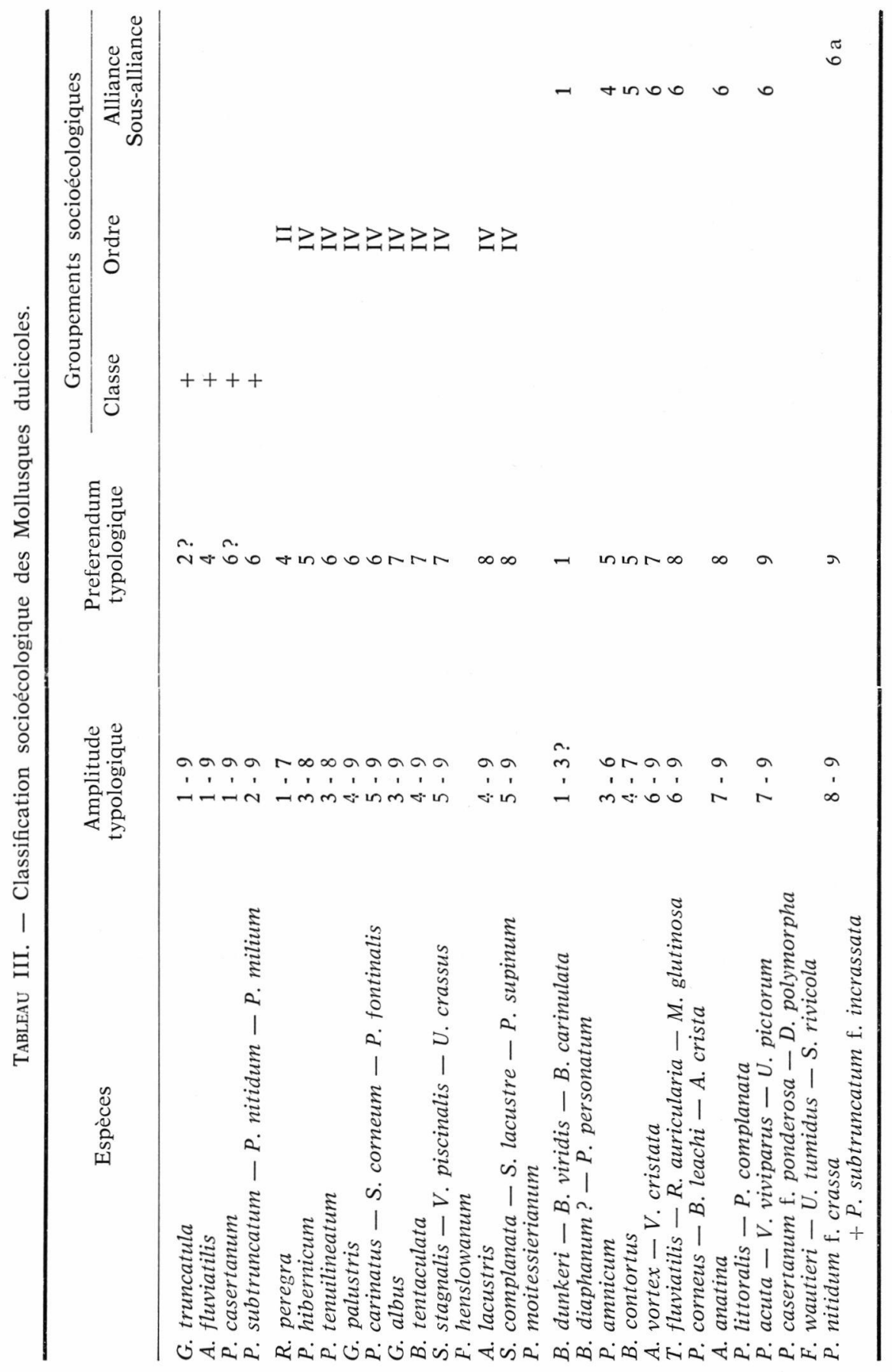


Nota: certaines espèces très peu représentées dans les prélèvements effectués n'ont pas été classées dans ce tableau. Il s'agit de $H$. minuta, A. spirorbis, S. nitida, P. obtusale, $P$. pulchellum, $P$. jenkinsi, en ce qui concerne cette dernière, il semble, d'après des données recueillies sur le Gapeau, la Seille... que son amplitude typologique s'étende des niveaux 4 à 9 .

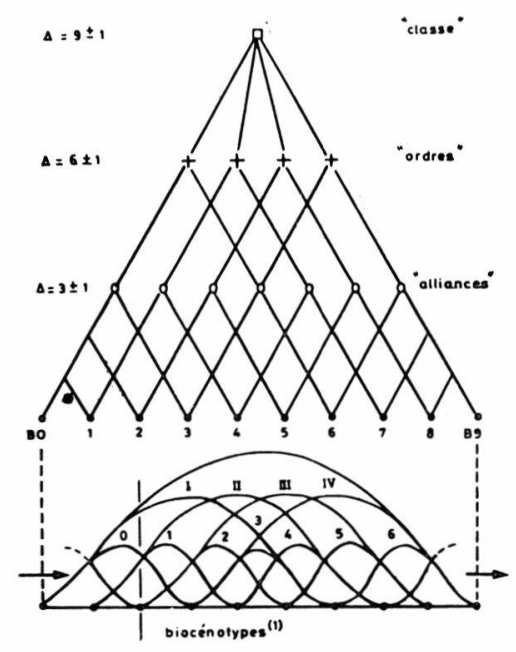

FIG. 5: Organisation des groupements "socio-écologiques" (d'après Verneaux, $197($ b).

\section{6. - EVOLUTION LONGITUDINALE \\ ET SIGNIFICATION TYPOLOGIQUE DES MOLLUSQUES COMPAREES A 4 GROUPES FAUNISTIQUES}

Les courbes d'évolution longitudinale de la richesse spécifique des 5 groupes faunistiques considérés (fig. 6 a) présentent une série de maxima se succédant dans un ordre typologique croissant pouvant "donner une image globale, simplifiée, de la composition faunistique d'un écosystème théorique " (Verneaux 1973). Comme celui des Téléostéens, le preferendum écologique global des Mollusques dulcicoles se situe au niveau du Potamon (niveau 8).

Ce type de distribution que l'on peut s'attendre à retrouver également chez les Odonates, les Hémiptères et la plupart des familles de Coléoptères, est quasi générale à l'intérieur de l'Embranchement des Mollusques si l'on excepte les pisidies qui atteignent leur diversité optimum au niveau 6 (fig. 6 b). On note par ailleurs que l'ordre des Basommatophores (Pulmonés) comprend le plus grand nombre d'espè- 
ces et que chez les Prosobranches un second maximum, d'amplitude plus réduite, se manifeste dans le premier niveau typologique.

Toutefois la présence de Gastéropodes et de Bivalves, dans les sources, n'est pas constante ; celles de type rhéocrène par exemple, en sont fréquemment dépourvus.

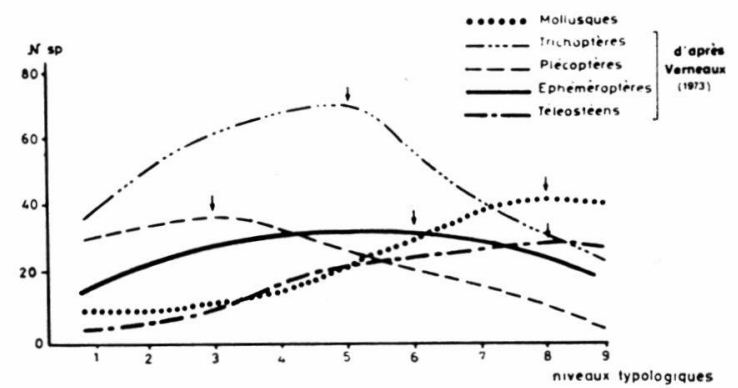

Fig. 6 a. - Evolution longitudinale de la richesse spécifique des Mollusques dulcicoles comparée à différents ordres faunistiques.

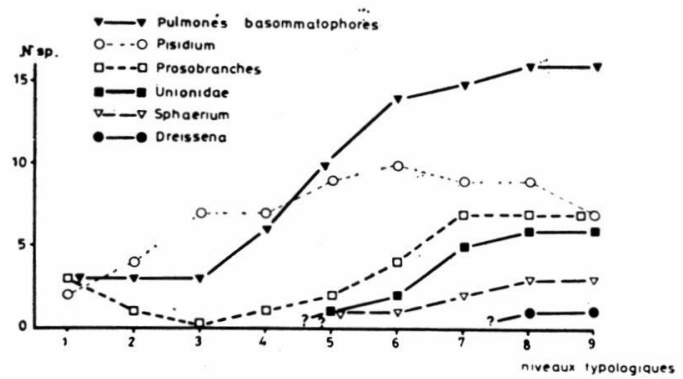

Fig. 6b. - Distribution longitudinale de la richesse spécifique des principaux groupes de Mollusques dulcicoles.

Les Mollusques, en comblant l'important déficit d'espèces laissé au niveau du Potamon par les Plécoptères, Trichoptères, Ephéméroptères, (tableau IV), dont les preferendum globaux appartiennent au rhithron, vont contribuer à l'élargissement de la gamme des groupements " socioécologiques " proposés par Verneaux (1976 b), permettant la détermination de l'appartenance typologique d'une station à partir de son peuplement, et la définition à des dimensions restreintes (mosaïques d'habitats et de biocénoses) d'associations et de sous-associations d'espèces. 
TableaU IV. - Nombre d'espèces recensées par niveau typologique.

\begin{tabular}{llrrrrrrrrr}
\hline & Niveaux typologiques & 1 & 2 & 3 & 4 & 5 & 6 & 7 & 8 & 9 \\
\hline Nombre & Plécoptères - Trichoptères & & & & & & & & & \\
d'espèces & Ephéméroptères - Poissons & 83 & 115 & 131 & 151 & 151 & 130 & 115 & 95 & 68 \\
\hline & $i d .+$ Mollusques & 91 & 123 & 140 & 165 & 174 & 160 & 153 & 137 & 109 \\
\hline
\end{tabular}

A l'exception des sources dont le peuplement malacologique comprend des espèces à répartition géographique limitée (cas des régions karstiques), la plus grande partie des Gastéropodes et Lamellibranches dulcicoles est largement distribuée dans tout le secteur prospecté et dans toute la France (Germain 1931 ; Kuiper 1966 et données personnelles sur le bassin de la Dordogne, de la Somme, de la Seine, de la Saône, de la Loire et du Gapeau : Var) si l'on considère le nombre d'espèces communes aux cours d'eau étudiés comparés deux à deux (Tableau V).

TableaU V. - Nombre d'espèces communes des cours d'eau étudiés comparés deux à deux.

\begin{tabular}{|c|c|c|c|c|c|c|c|c|c|}
\hline 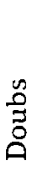 & $\stackrel{8}{3}$ & $\begin{array}{l}\underset{0}{0} \\
\underset{0}{0} \\
0\end{array}$ & 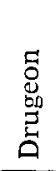 & 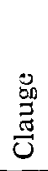 & 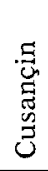 & 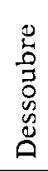 & 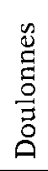 & 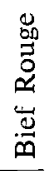 & \\
\hline \multirow[t]{9}{*}{49} & 39 & 41 & 19 & 22 & 9 & 10 & 8 & 4 & Doubs \\
\hline & \multirow[t]{8}{*}{42} & 33 & 19 & 22 & 9 & 10 & 8 & 4 & Aube \\
\hline & & \multirow[t]{7}{*}{41} & 17 & 20 & 9 & 10 & 8 & 4 & Ognon \\
\hline & & & \multirow[t]{6}{*}{23} & 13 & 8 & 8 & 6 & 4 & Drugeon \\
\hline & & & & \multirow[t]{5}{*}{23} & 8 & 9 & 8 & 3 & Clauge \\
\hline & & & & & \multirow[t]{4}{*}{12} & 9 & 7 & 4 & Cusançin \\
\hline & & & & & & 10 & 8 & 4 & Dessoubre \\
\hline & & & & & & & 8 & 3 & Doulonnes \\
\hline & & & & & & & & $\overline{4}$ & Bief Rouge \\
\hline
\end{tabular}

Capables d'intégrer des situations écologiques fort diverses, ce qui constitue une expression de leur degré d'euryécie générale déjà souligné par Hubendick (1958) pour les Gastéropodes et par Wolff (1968) pour les Unionidae, les Mollusques se situent entre les Ephéméroptères et les Poissons en ce qui concerne cette faculté d'adaptation.

En outre, le faible nombre d'espèces recensées en France, comparé à d'autres groupes d'Invertébrés (Trichoptères, Coléoptères...) et la 
vaste répartition de la plupart d'entre elles permettra sans doute d'aboutir à une généralisation rapide du modèle proposé après l'inventaire d'autres bassins et de cours d'eau côtiers.

\section{CONCLUSIONS}

L'application de l'analyse factorielle des correspondances aux données recueillies dans 9 cours d'eau morphologiquement très différents, a permis d'obtenir une hiérarchisation écologique des espèces s'organisant en un "continuum a noda ». Après confrontation aux données typologiques stationnelles, il a été possible de rapporter les 11 groupements aux 10 niveaux proposés par Verneaux (1973).

La structure typologique définie permet d'avoir une vision globale et synthétique de la distribution théorique des espèces de Mollusques le long d'un écosystème d'eau courante ; elle constitue le cadre général dans lequel s'inscrit l'écologie de ce groupe, indispensable à l'interprétation écologique de peuplements de Mollusques d'une station ou d'un écosystème d'eau courante dans lequel ils peuvent être replacés.

Le caractère lénitophile et potamobionte de la plupart des espèces constitue, comme en témoigne l'évolution longitudinale de la richesse spécifique des Mollusques, l'une des originalités de ce groupe faunistique. Dans le cadre, plus général, des recherches synécologiques visant notamment à l'établissement d'une biotypologie intégrant l'ensemble des organismes aquatiques, les Mollusques vont contribuer à l'élargissement de la gamme des groupements "socio-écologiques" proposés par Verneaux (1976 b). C'est à partir de celle-ci que pourra être élaboré un système de référence permettant aux services d'application de disposer d'un outil de travail répondant aux besoins actuels : critières et objectifs de qualité, études d'impact et d'aménagements...

\section{REMERCIEMENTS}

Je remercie M. J. G. J. Kuiper qui a bien voulu vérifier toutes mes déterminations de Pisidies et MM. les Professeurs J. Verneaux et M. Lamotte pour les conseils qu'ils m'ont prodigués lors de l'élaboration de ce travail. 


\section{TRAVAUX CITÉS}

Adam (W.). 1942. - Sur la répartition et la biologie de Hydrobia jenkinsi Smith en Belgique. Bull. Mus. r. Hist. nat. Belg., 18 : 1-18.

Boycotr (A. E.). 1936. - The habitats of the freshwater Mollusca in Britain. $J$. Anim. Ecol., 5 : 116-186.

Calas (P.). 1954. - Précisions sur l'extension en France du genre Gundlachia Pfeiffer. Bull. mens. Soc. Linn. Lyon., 23 : 193-194.

CoRdier (B.). 1965. - L'analyse factorielle des correspondances. Thèse $3^{\text {e }}$ cycle Univ. Rennes, $66 \mathrm{p}$.

Duvigneaud (P.). 1946. - La variabilité des associations végétales. Bull. Soc. Rev. Bot. Belg., 58 : 107-134.

FAVRE (J.). 1927. - Les Mollusques post-glaciaires et actuels du bassin de Genève. Mém. soc. Phys. et Hist. natur. Genève, 40 (3) : 171-434.

Germain (L.). 1931. - Mollusques terrestres et fluviatiles. In Faune de France. 21-22 Lechevalier éd., Paris, 897 p.

Geyer (D.). 1911. - Die Molluskenfauna des Neckars. Jh. Ver. Vaterl. Naturk. Württemberg, 354-371.

Guinocher (M.). 1973. - Phytosociologie. Collection d'Ecologie. I. Masson éd., Paris, $227 \mathrm{p}$.

HeYwood (J.) et Edwards (R. Q.). 1962. - Some aspects of the ecology of Potamopyrgus jenkinsi Smith. J. Anim. Ecol., 31 (2) : 239-250.

HubeNDicK (B.). 1958. - Factors conditioning the habitat of freshwater snails. Bull. Org. Mond. Santé, 18 : 1072-1080.

Huet (M.). 1949. - Aperçu des relations entre la pente et les populations piscicoles dans les eaux courantes. Schweiz. Z. Hydrol., 11 (3-4) : 332-351.

Illies (J.) et Botosaneanu (L.). 1963. - Problèmes et méthodes de la classification et de la zonation écologique des eaux courantes, considérées surtout du point de vue faunistique. Mitt. internat. Verein. Limnol., $12: 1-57$.

Kuiper (J. G. J.). 1966. - La distribution des espèces vivantes du genre Pisidium C.P.F. en France. J. Conchyol., 105 (4) : 181-215.

Kuiper (J. G. J.) et WolfF (W. J.). 1970. - The Mollusca of the estuarine region of the rivers Rhine, Meuse and Scheldt in relation to the hydrography of the area. III. The genus Pisidium. Basteria, $34: 1-42$.

Masonie (J. P.), Mathieu (D.) et Wirber (J. C.). 1971. - Application de 1'analyse factorielle à l'étude des paysages. Cah. Géogr. Besançon, 4 (9) : 1-51.

MeIER-BrooK (Von C.). 1970. - Untersuchungen zur Biologie einiger Pisidium Arten (Mollusca, Eulamellibranchiata. Sphaeriidae). Arch. Hydrobiol., suppl., $38(1-2): 73-150$.

Mouthon (J.). 1979. - Structure malacologique de la rivière Aube. Annls Limnol., 15 (3) : $299-315$.

Mouthon (J.). 1980. - Contribution à l'écologie des Mollusques des eaux courantes - Esquisse biotypologique et données écologiques. Thèse $3^{c}$ cycle, Univ. Paris VI, $169 \mathrm{p}$.

Odum (E.). 1976. - Ecologie. Traduction deuxième édition. R. Bergeron. HRW Ltée (éd.), Montréal, $254 \mathrm{p}$.

SAcchi (C. F.) et TestaRd (P.). 1971. - Ecologie animale - Organismes et milieu. Doin éd., Paris : $480 \mathrm{p}$.

VERNEAUX (J.). 1973. - Cours d'eau de Franche-Comté (Massif du Jura) - Recherches écologiques sur le réseau hydrographique du Doubs - Essai de biotypologie. Thèse Doct. ès Sci. Nat. Sci. Univ. Besançon, $257 \mathrm{p}$.

VerneauX (J.). 1976 a. - Biotypologie de l'écosystème " eau courante ". La structure biotypologique. C. R. Acad. Sc. Paris, $283:$ 1663-1666. 
Verneaux (J.). 1976 b. - Biotypologie de l'écosystème « eau courante ». Les groupements socio-écologiques. C. R. Acad. Sc. Paris, 283 : 1791-1793.

Verneaux (J.). 1977 a. - Biotypologie de l'écosystème « eau courante ». Déterminisme approché de la structure biotypologique. C. R. Acad. Sc. Paris, 284 : 77-79.

Verneaux (J.). 1977 b. - Biotypologie de l'écosystème «eau courante ». Détermination approchée de l'appartenance typologique d'un peuplement ichtyologique. C. R. Acad. Sc. Paris, 284 : 675-678.

Verneaux (J.). 1980. - Fondements biologiques et écologiques de l'étude de la qualité des eaux continentales - Principales méthodes biologiques in: Pesson. La pollution des eaux continentales. Incidence sur les biocénoses aquatiques. Gauthier-Villars éd. : 289-345.

WALTER (J. E.) et KuIPER (J.G. J.). 1978. - Über Verbreitung und ökologie von Sphaeriiden im Zurichsee (Mollusca: Eulamellibranchiata). Schweiz. Z. Hydrol., 40 (1) : 60-86.

WoLfF (W. F.). 1968. - The Mollusca of the estuarine region of the rivers Rhine, Meuse and Scheldt in relation to the hydrography of the area. I. The Unionidae. Basteria, 32 : 13-47.

ZhadiN (V. J.). 1931-1935. - Über die ökologische und geographische Verbreitung des Süsswassermollusken in der U. d. S.S.R. Zoogeographica, 2 : 295-554. 